\title{
Atomic Force Microscopy of Tissue Sections \\ Is a Useful Complementary Tootin Biomedical \\ Morphological Studies
}

DOI: 10.17691/stm2018.10,1.09

Received October 19, 2017

P.S. Timashev, DSc, Director of the Institute for Regenerative Medicine'; Leading Researcher'; Researcher ${ }^{3}$;

A.V. Koroleva, PhD, Researcher, Nanotechnology Unit";

N.A. Konovalov, MD, DSc, Head of the Spinal Neurosurgery Department5;

S.L. Kotova, PhD, Senior Researcher, Laboratory of Modified Polymer Systems,

Department of Polymers and Composites; ${ }^{3}$ Senior Researcher 6 ;

A.B. Solovieva, DSc, Head of the Laboratory of Modified Polymer Systems, Department of Polymers and Composites ${ }^{3}$

I.M. Sechenov First Moscow State Medical University, $8 / 2$ Trubetskaya St., Moscow, 119991, Russia;

2Institute of Photonic Technologies, Research Center "Crystallography and Photonics",

Russian Academy of Sciences, 2 Pionerskaya St., Moscow, Troitsk, 142190, Russia;

${ }^{3}$ Semenov Institute of Chemical Physics, Russian Academy of Sciences, 4 Kosygina St., Moscow,

119991, Russia;

${ }^{4}$ Laser Zentrum Hannover, 8 Hollerithallee, Hannover, 30419, Germany;

${ }^{5}$ N.N. Burdenko National Scientific Center for Neurosurgery, 16, 4th Tverskaya-Yamskaya St., Moscow,

125047, Russia;

${ }^{6}$ Federal Research Clinical Center, Federal Medico-Biological Agency of Russia, 28 Orekhovy Blvd., 115682,

Moscow, Russia

The aim of the study was to demonstrate a good diagnostic potential of atomic force microscopy (AFM) in tracking morphological changes in the extracellular matrix (ECM) of connective tissue due to pathological processes. Here we summarize our experience in AFM application in a number of biomedical studies on the connective tissue disease, both for the research and clinical purposes.

Materials and Methods. Depending on the project application (experimental or clinical), the tissue specimens were harvested either from animals, or from patients in the course of their surgical treatment, or post mortem.

AFM images of fixed tissue slices on glass slides were acquired with a Solver P47 AFM instrument (NT-MDT, Russia), in the semicontact mode. For mechanical properties mapping, the images were acquired on air in the PeakForce Quantitative Nanomechanical Mapping mode (PeakForce QNM ${ }^{\circledR}$ ), using a MultiMode 8 atomic force microscope (Bruker, USA). The regions of interest for scanning were selected in accordance with the histological assignments for the same sample, based on the view of a sample in the built-in optical microscope of the AFM instrument setup. To quantify the changes in the ECM morphology visualized by AFM imaging, we applied flickernoise spectroscopy parameterization.

Results. AFM has been shown to reveal visible deviations from the normal morphology of the ECM in diseased tissues. We found that AFM and related techniques are capable of tracking disease-related changes at different levels of collagen organization in the ECM. At the microscale, AFM may detect loosening and disorganization of collagen fibers (e.g., in a dysplastic process), or the opposite process of their packing into tight parallel bundles in a fibrotic process. AFM may also monitor the ratio between collagen and non-fibrous material of the ECM, for example, in inflammatory and neoplastic processes. At the level of collagen fibrils, AFM may reveal early signs of the matrix destruction and remodeling not visible at the microscopic level. The flicker-noise spectroscopy parameters provide quantification of the morphological changes visualized by AFM. The PeakForce QNM ${ }^{\circledR}$ and nanoindentation studies give a further insight into the state of ECM via tracking changes in the local mechanical and adhesive properties.

All our AFM studies appeared in a good agreement with the histological findings and generally had a superior sensitivity to pathologyrelated ECM rearrangements.

Conclusion. AFM may serve as a valuable complementary diagnostic tool for tracking pathological changes in the connective tissue.

Key words: atomic force microscopy; extracellular matrix; collagen; connective tissue; flicker-noise spectroscopy; nanomechanics; PeakForce QNM ${ }^{\circledR}$.

How to cite: Timashev P.S., Koroleva A.V., Konovalov N.A., Kotova S.L., Solovieva A.B. Atomic force microscopy of tissue sections is a useful complementary tool in biomedical morphological studies. Sovremennye tehnologii v medicine 2018; 10(1): 70, https://doi.org/10.17691/ stm2018.10.1.09

Corresponding author: Peter S. Timashev, e-mail: timashev.peter@gmail.com 


\section{Introduction}

Connective tissue has always attracted attention as an object for ultramicroscopy studies due to a very high content of extracellular matrix (ECM) which often exceeds the cell content. The primary component of the connective tissue ECM is collagen forming intricate fibrous structures, besides, ECM contains elastic fibers and non-fibrous gellike ground substance, consisting of glycosaminoglycans, proteoglycans, and glycoproteins [1]. To sustain the mechanical integrity and strength of connective tissue, collagen is arranged into a complex hierarchy from the molecular scale (tropocollagen) to the nanoscale (collagen fibrils), microscale (collagen fibers and their bundles) and up to the macroscale (e.g., whole ligaments). The staggered arrangement of collagen molecules at the lowest level of its packing (with the stagger distance of about $67 \mathrm{~nm}$ ) is precisely preserved to the higher organization levels and is known as the D-period of collagen [2]. The characteristic banding pattern of collagen fibrils originated from the D-period is easily observed via electron microscopy and atomic force microscopy (AFM).

In spite of the recent development of laser microscopic techniques for the collagen studies, such as laser scanning microscopy and optical coherence tomography $[3,4]$, conventional histological techniques remain by far the most widely used methods of connective tissue investigation both in the experimental and clinical setting. Stained histological sections provide the majority of information about the overall appearance of the collagen matrix, elastic fibers, and cells. However, optical microscopy is unable to reveal the inner arrangement of collagen structures, such as packing of collagen fibrils inside fibers nor is it capable of gaining information on the local material properties of collagenous structures. Therefore, AFM may become a powerful tool to complement current optical microscopy modalities, since it provides morphological information on the ECM structure at the microscale, nanoscale and beyond, and may also provide additional information on the local mechanical and adhesive properties of the ECM.

In the last years, AFM as a technique has been extensively developed in accordance with its growing application in biology and biophysics (see, e.g., a review by Maver et al. [5]). Not only microscopes have become essentially more suitable for the specifics of biological samples, but also new approaches in the technique itself, such as PeakForce $\mathrm{QNM}^{\circledR}$ by Bruker (USA), allow unprecedented resolution combined with measurements of physical properties for cells, tissues, and materials created for biomedical application. Thus, the recent developments have turned AFM from a mainly imaging tool to a powerful physical instrument with various modalities.

The characteristic packing of collagen fibrils and fibers was obtained for such types of connective tissue as skin, intervertebral disk [6], cartilage [7, 8], tendon [9], bone
[10], vocal folds [11], sclera [12] and cornea [13], vaginal wall tissue [14]. Nanomechanical studies have also been proven quite informative in the ECM assessment [15, 16]. In our studies, we set a goal of developing an AFMbased approach for combined biomedical morphological studies, in which standard histological tissue sections may be used, and one and the same sample may be studied interchangeably by optical microscopy and AFM techniques. In the course of our previous studies [17, 18], we have shown that AFM studies of dry histological sections on air may be successfully used for tracking pathological alterations of the collagen hierarchical structure in the ECM of various tissues.

The aim of the study was to demonstrate a good diagnostic potential of atomic force microscopy and related techniques (nanoindentation, quantitative nanomechanical mapping, flickernoise patarameterization of AFM images) in tracking morphological changes in the extracellular matrix of connective tissue due to pathological processes. Such a study may become a basis for a convenient screening method applied as a complementary tool to the conventional histological analysis.

\section{Materials and Methods}

Preparation of samples. According to the purposes of each biomedical study, the samples were harvested either as surgical biopsies (studies of pelvic organ prolapse [17], chondrosarcoma, congenital chest wall deformities), or post-mortem (study of coronary arteries affected by atherosclerosis [18]), or from experimental animals (study of radiation damage of pelvic organs). The human and animal studies were performed in accordance with the corresponding international laws on the legal and ethical norms and in each case were approved by a local Ethical Committee.

The general procedure for preparation of histological sections suitable for AFM studies was as follows. Segments of tissue were fixed in $10 \%$ neutral formalin for $48 \mathrm{~h}$, dehydrated in a graded series of alcohol, embedded in paraffin wax and cut into 5-7 $\mu \mathrm{m}$ serial sections for the histological and AFM studies, respectively. For the histological studies, the paraffin slices were stained with hematoxylin and eosin or with van Gieson's picrofuchsin, embedded in the mounting media and covered by coverslips. For AFM studies, the sections were thoroughly deparaffinized, left unstained and uncovered.

To minimize the "processing artifacts" from the samples' drying and formalin fixation [19, 20], the same preparation protocol was maintained for all the tissue sections in either the histological or AFM study.

Atomic force microscopy imaging study. Generally, AFM images of the fixed tissue sections on glass slides were acquired with a Solver P47 AFM instrument (NTMDT, Russia), in the semi-contact mode, using a precision Solver scanner $(14 \times 14 \times 2 \mu \mathrm{m})$ and silicon TESP probes (Bruker, USA). General-view $14 \times 14 \mu \mathrm{m}$ images and 
detailed $6 \times 6$ and $3 \times 3 \mu \mathrm{m}$ topography and phase images were obtained at a scan rate of $1 \mathrm{~Hz}$ and a $512 \times 512$ pixels resolution.

The regions of interest (ROI) for scanning were selected in accordance with the histological assignments for the same sample, based on the view of a sample in the built-in optical microscope of the AFM instrument setup (Figure 1). At least 10 images from different regions of each section were acquired. For visualization, AFM images underwent standard processing (flattening and cleaning) with the image processing software Nova v. 1.0.26.1443 (NT-MDT, Russia). Raw images without any processing were used in the flicker-noise spectroscopy (FNS) computations.

Flicker-noise spectroscopy for atomic force microscopy images. For the quantitative assessment of the changes in the ECM morphology visualized by AFM imaging, we applied a FNS parameterization technique [21]. In the FNS approach, the roughness profiles $h(x)$ in every $y$-line in an AFM image are considered as a series of chaotic signals (dynamic variables), and the FNS technique seeks correlations in these series. The derived surface FNS nanometrology parameters are related to the autocorrelation function for every profile. The FNS power spectra allow selection of different ranges of spatial frequencies in the $h(x, y)$ profiles. The low-frequency ("resonant") range (which mostly reflects major bends of the surface originating from the macro-manipulations such as sample positioning) is disregarded. The highfrequency range is described in the terms of two basic types of sharp irregularities. The first type - "jumps" represent relatively moderate-height steps against the low-frequency baseline. The second type - "spikes" are single intense bursts of the signal. Each of these two types of irregularities is ascribed a corresponding FNS parameter: a "stepwiseness factor" $\sigma(\mathrm{nm})$ for the jump-like irregularities and a "spikiness factor" $S\left(L_{0}{ }^{-1}\right)$ $\left(n m^{2} \mu \mathrm{m}\right)$ for the spike-like irregularities (where $L_{0}$ is the

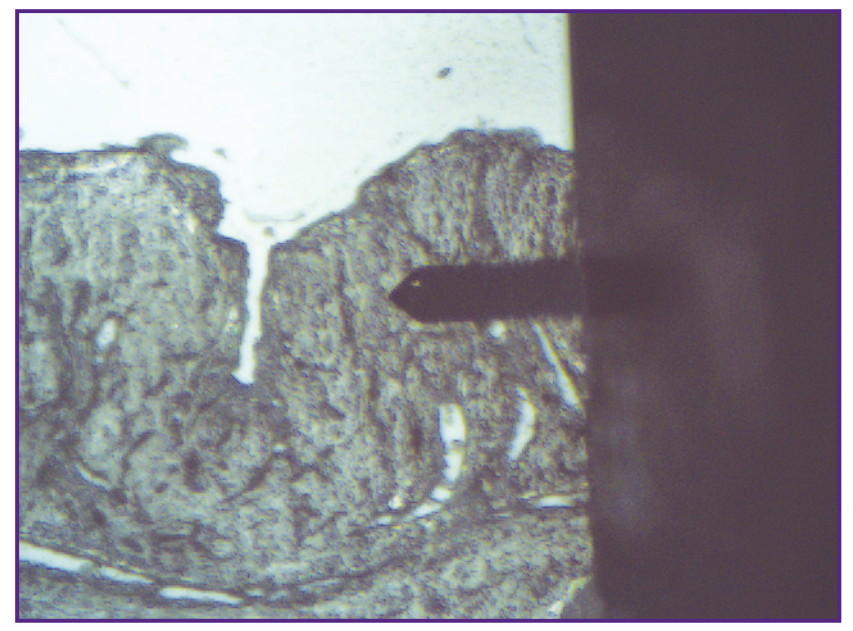

Figure 1. Optical microscopy image of a rat bladder section with the atomic force microscopy tip in contact length of correlation for the nanoscale high-frequency irregularities in the profile). There exist also other FNS parameters, which are of a relatively minor importance for AFM images. The entire FNS procedure, including the derivation and calculation of the "stepwiseness factor" $\sigma$ and the "spikiness factor" $S\left(L_{0}^{-1}\right)$ is presented in [21].

We used the two above FNS parameters "stepwiseness factor" $\sigma$ and the "spikiness factor" $S\left(L_{0}^{-1}\right)$ - to quantitatively characterize the visualized surface morphology of the ECM. The FNS computations were performed according to the algorithm described in [21] using a custom-made software.

Nanomechanical studies. The mechanical properties (hardness and Young's modulus) of specimens were studied either by nanoindentation or using the PeakForce QNM $^{\circledR}$ (Bruker, USA) mode.

Nanoindentation studies. Nanoindentation was performed using a nanosclerometry/nanoindentation block of the NTEGRA-Therma AFM instrument (NTMDT, Russia) and the NanoScan software (NT-MDT, Russia). The block consisted of a nanosclerometry/ nanoindentation head with a displacement detector, a piezoresonance probe and a diamond Berkovich indenter tip. The ranges of the measured hardness were $0.1-80 \mathrm{GPa}$, and the ranges for the Young's modulus were 0.1-3000 GPa. 200 indents per sample, on average, were made. A melted quartz sample with known hardness and Young's modulus values was used as a reference sample.

PeakForce QNM studies. Nanomechanical images of the tissue sections deposited on glass slides were acquired on air in the PeakForce Quantitative Nanomechanical Mapping mode (PeakForce QNM ${ }^{\circledR}$, Bruker, USA), using a MultiMode 8 atomic force microscope with a NanoScope $\mathrm{V}$ controller and $\mathrm{E}$ scanner (Bruker, USA). The regions of interest for AFM scanning were selected based on the histological assignment, according to the sample's view in the optical microscope combined with the AFM instrument. At least 5 images from different regions of each section were acquired.

AFM imaging was conducted with RTESPA- 150 probes (Bruker, USA) with a nominal spring constant of $5 \mathrm{~N} / \mathrm{m}$, nominal frequency of $150 \mathrm{kHz}$ and nominal tip radius of $8 \mathrm{~nm}$. The cantilevers' deflection sensitivities were measured in respect to the sapphire standard (Bruker, USA). The real spring constant of cantilevers was measured via the thermal tune method. The tip radius evaluation was performed by the relative method using a polystyrene standard (Bruker, USA).

General-view $12 \times 12 \mu \mathrm{m}$ images and detailed $3 \times 3 \mu \mathrm{m}$ images were obtained at a scan rate of $1 \mathrm{~Hz}$ and a $512 \times 512$ pixels resolution. The Height, Peak Force Error, Young's modulus (by the DTM model), adhesion and deformation channels were acquired simultaneously.

The raw AFM images were processed using the NanoScope Analysis software v. 1.10 (Bruker, USA).

Histological studies. The conventional histological studies via the van Gieson's or hematoxylin and eosin 
staining protocols were performed for assessment of the collagen structures and for the correct assignment of the ROI in the AFM study.

\section{Results}

Extracellular matrix microstructural alterations observed by atomic force microscopy. At the microscale level, the complex hierarchical structure of collagen in the ECM is represented by collagen fibers and their bundles. Our AFM studies of ECM in various pathological processes have shown that changes in the arrangement of collagen fibers are perfectly visualized by AFM at the scales from microns to tens of microns.

For example, AFM imaging has explicitly shown that the ECM of connective tissue (skin and pelvic ligament) undergoes a drastic rearrangement in a pathological dysplastic process leading to pelvic organ prolapse (POP). In particular, such morphological signs of the POP pathology have been differentiated as loosening and separation of collagen fibers in the fiber bundles, disintegration, and disordering of collagen fibers. In Figure $2(a)$, (b), these signs are clearly seen in overview $100 \mu \mathrm{m}$ AFM images of the pelvic ligament ECM.

The micrometer-scale AFM imaging allowed distinction between different stages of atherosclerosis, as well as distinction between stable and vulnerable (potentially dangerous) atherosclerotic plaques at the terminal stage of the plaque development. In Figure 2 (c), (d), the latter findings are presented, with the loose and heterogeneous packing of fibers in an unstable plaque (Type Va) vs a significantly tighter packing in a stable fibrotic plaque (Type Vc) due to the presence of amorphous material covering and gluing the collagen structures together.

In an AFM study of bone tumor development, we compared the morphology of chondrosarcoma of histologically malignant grades I-III. The AFM imaging showed a clear correlation between the content of the fibrous collagenous elements in the ECM of a bone tumor and the degree of its malignancy (Figure 2 (e), (f)).

The AFM imaging study applied to track post-radiation ECM changes of pelvic organs has revealed the initial destruction of the protein matrix and the following alterations in the packing of collagen fibers indicative of the onset of fibrotic processes. In Figure 2 (g), (h), the early postradiation fibrotic changes in the rectal ECM are presented in comparison with the intact tissue ECM. The rearrangement of collagen fibers from a meshwork appearance, with 1-2 $\mu$ m-thick fibers, into dense oriented structures of a significant thickness $(3-7 \mu \mathrm{m})$ is clearly visible.

Atomic force microscopy tracking the packing of collagen fibrils in the normal and pathologically altered tissue. To study the packing of collagen fibrils inside fibers, their thickness and the inner structure of fibrils, we obtained high-resolution topography and
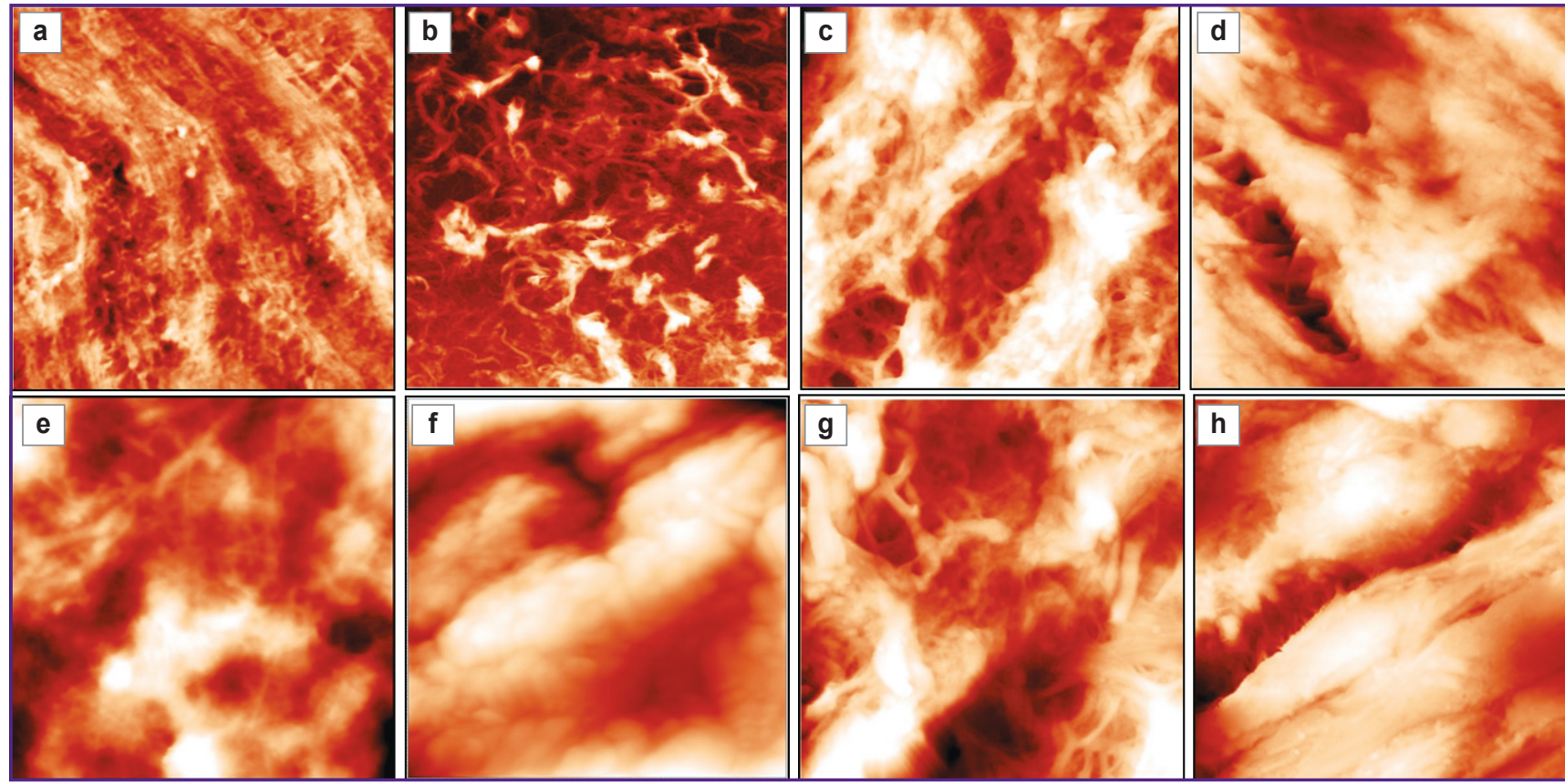

Figure 2. Overview atomic force microscopy images demonstrating disease-related microstructural extracellular matrix features (collagen fibers and their bundles):

(a), (b) normal pelvic ligament vs pelvic ligament in pelvic organ prolapse; (c), (d) atherosclerotic lesion Type Va vs atherosclerotic lesion Type Vc; (e), (f) chondrosarcoma grade I vs grade III; $(g)$, (h) normal rectum extracellular matrix vs extracellular matrix showing signs of early post-radiation fibrosis (2 Gy, 1 month post-irradiation). The scan sizes are $100 \times 100 \mu \mathrm{m}(\mathrm{a})$, (b) and $14 \times 14 \mu \mathrm{m}(\mathrm{c})-(\mathrm{h})$ 
phase images. As shown by the following examples, the structure of collagen fibrils is also sensitive to pathological processes in the connective tissue.

In the study of the ECM in POP, AFM imaging has shown that collagen fibers of the normal skin are formed by bundles of quasi-parallel tightly packed fibrils, while in the POP group, the chaotic interweaving of fibrils and bundles of fibrils prevails (Figure 3 (a), (b)). In the experimental group, collagen fibrils are also frequently deformed, fragmented and shortened as compared to the healthy control.

High-resolution imaging has also been used to examine the details of collagen packing in the ECM of developing atherosclerotic plaques. Figure 3 (c), (d) demonstrates a striking difference between vulnerable (Type Va) and stable (Type Vc) plaques due to a presence of a large amount of a non-fibrous material covering collagen fibrils in a stable plaque. The sticky non-fibrous material provides a tighter packing of fibrils and fibers and thus mechanical stability for this type of atherosclerotic lesions, as opposed to loosely packed collagen structures in a vulnerable plaque responsible for its instability and propensity to rupture.

The radiation-induced ECM destruction in the bladder and rectum is significant at the level of collagen fibrils, as demonstrated by high-resolution AFM imaging. With the

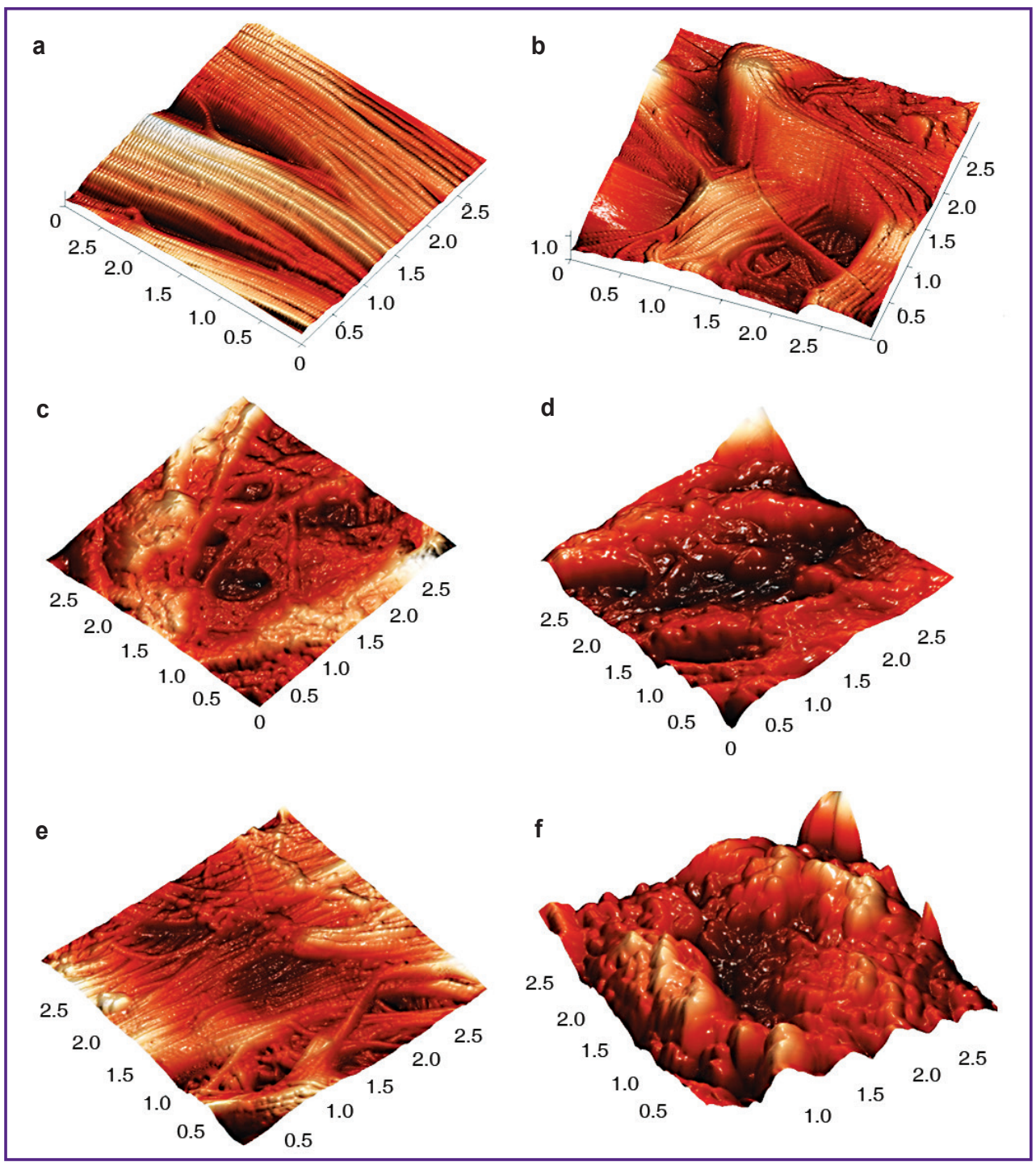

Figure 3. High-resolution 3D atomic force microscopy images demonstrating alteration of the extracellular matrix nanostructure (packing of collagen fibrils) caused by pathological processes:

(a), (b) normal perineal skin vs skin in pelvic organ prolapse; (c), (d) atherosclerotic lesion Type Va vs atherosclerotic lesion Type Vc; (e), (f) normal rectum extracellular matrix vs extracellular matrix undergoing destruction after a high-dose irradiation (22 Gy, 1 day post-irradiation). The scan sizes are $3 \times 3 \mu \mathrm{m}$ 


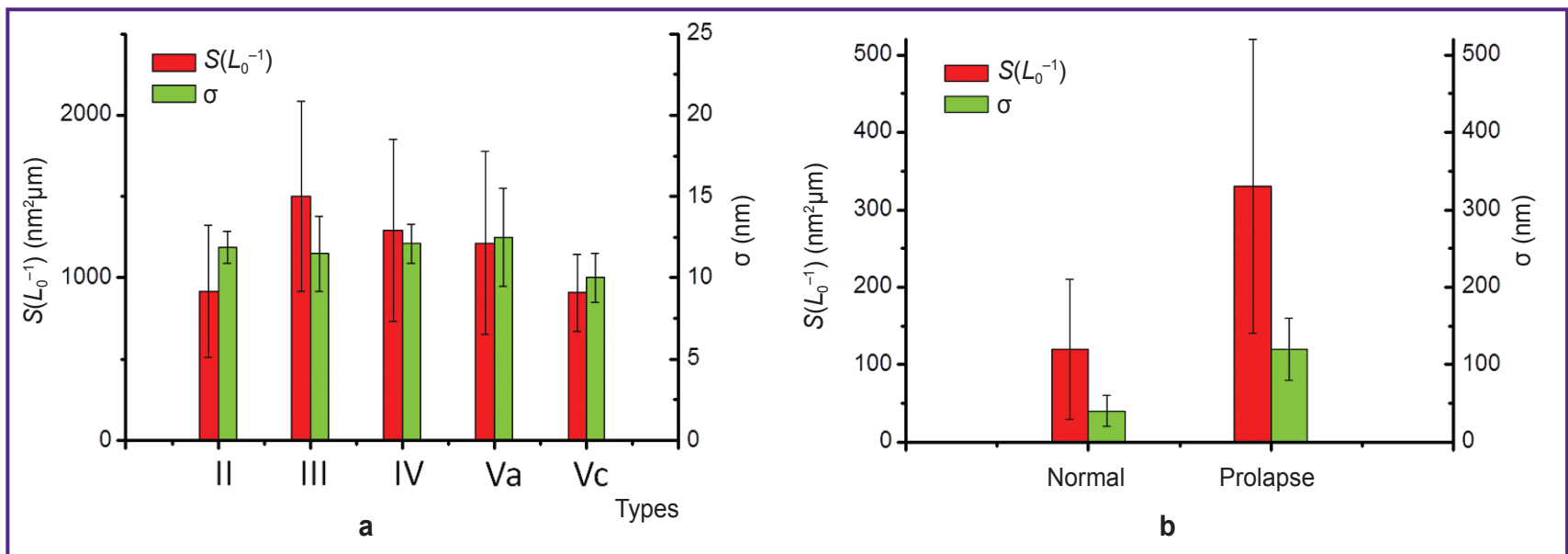

Figure 4. Flicker-noise spectroscopy parameterization of atomic force microscopy images obtained from the extracellular matrix:

(a) atherosclerotic plaques of different clinical types $(6 \times 6 \mu \mathrm{m}$ scans) and (b) normal skin and skin of pervic organ prolapse patients $(3 \times 3 \mu \mathrm{m}$ scans $)$

radiation dose increase, the collagen fibrils tend to thicken and lose their initial local tight quasi-parallel arrangement, forming a rather disordered structure. The complete loss of tight fibrils' packing in the rectum ECM and a complete coverage of collagen fibrils with a thick continuous layer of unstructured proteinaceous material after a high radiation dose is demonstrated in Figure $3(\mathrm{e})$, (f).

Quantification of the extracellular matrix morphological changes by flicker-noise spectroscopy. To quantify the observed differences in the ECM morphology, we routinely applied the FNS parameterization technique for AFM images. The FNS numerical data frequently provide additional valuable information on the ECM changes, along with the visual analysis of AFM images.

In Figure 4, two examples of FNS application for quantification of AFM topography images are presented. In Figure 4 (a), the results of parameterization of a large number of AFM images - the two major FNS parameters, "stepwiseness factor" $\sigma$ and "spikiness factor" $S\left(L_{0}{ }^{-1}\right)$ - show visible distinctions in the morphology of atherosclerotic plaques of various types, from the early (Type II) to advanced stages of atherosclerosis (Types IV and V). In Figure 4 (b), a notable increase of both FNS parameters is detected in the POP group as compared to the control group.

In Figure 5, the displayed plots of the FNS parameters vs radiation dosage demonstrate a universal character of morphological changes occurring post-irradiation in the connective tissue of bladder, rectum and skin: the initial growth of the FNS parameters associated with the structure disordering followed by the later decrease (surface smoothening) related to the accumulation of nonfibrous material.

Nanomechanical properties of collagen structures by atomic force microscopy-related techniques. Nanoindentation is a technique for determination of the local nanomechanical properties of a material, closely related to AFM. Using the nanoindentation technique, we measured the nanomechanical properties of the ECM for clinical samples in the POP study. The values of hardness and Young's modulus of the fibril bundles appeared essentially reduced in the skin samples from the POP group, as compared to the corresponding values in the control group (Figure 6).

The nanomechanical properties may also be measured in a special AFM mode developed by Bruker, named a PeakForce Quantitative Nanomechanical Mapping mode (PeakForce $\mathrm{QNM}^{\circledR}$ ). PeakForce $\mathrm{QNM}^{\circledR}$ allows mapping the Young's modulus and adhesive properties along with the topography during one and the same scanning procedure. The information which may be acquired in the QNM mode is presented in Figure 7, by the example of the rib cartilage ECM. Two types of collagen fibrils' packing are shown - the native ECM fibril network and the packing which is characteristic of amianthoid transformation of the rib cartilage. The drastic difference in the ECM nanomorphology visible in the topography images (a) and (d) is further detailed in the Young's modulus map (b) and (e) demonstrating the solid parallel packing of collagen fibrils in the amianthoid transformation-altered ECM, as compared to the absence of order in the arrangement of fibrils inherent for the native ECM. The map of adhesive properties (c) and (f) shows spots of the increased adhesion (marked with arrows) corresponding to sites of glycosaminoglycans accumulation. As seen from Figure 7 (c), (f), their random distribution in the native ECM is substituted by localization between the bundles of parallel collagen fibrils in the amianthoid transformationaltered ECM. The Young's moduli of the ECM averaged over a number of PeakForce $\mathrm{QNM}^{\circledR}$ scans showed that the normal rib cartilage ECM is characterized by higher 

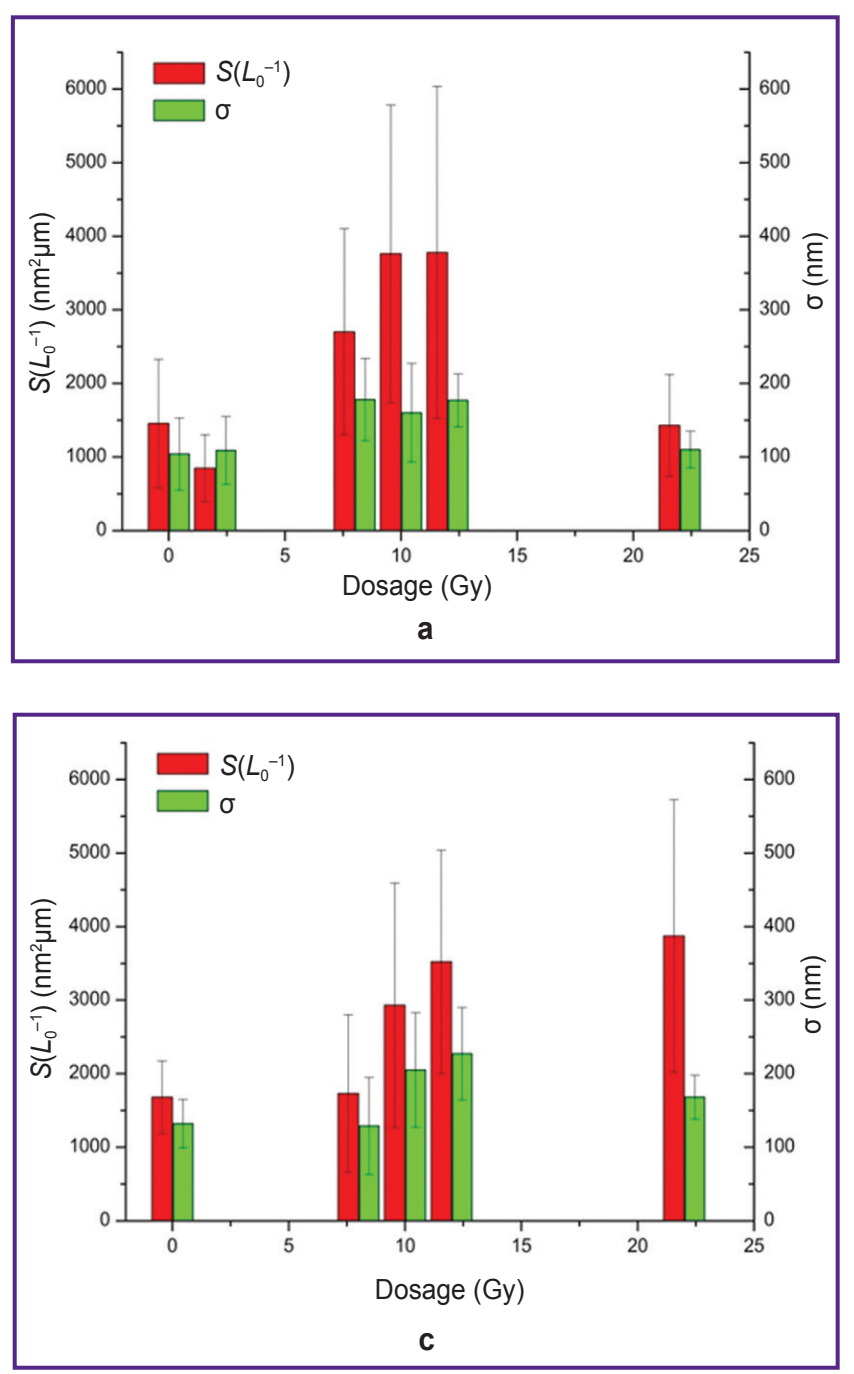

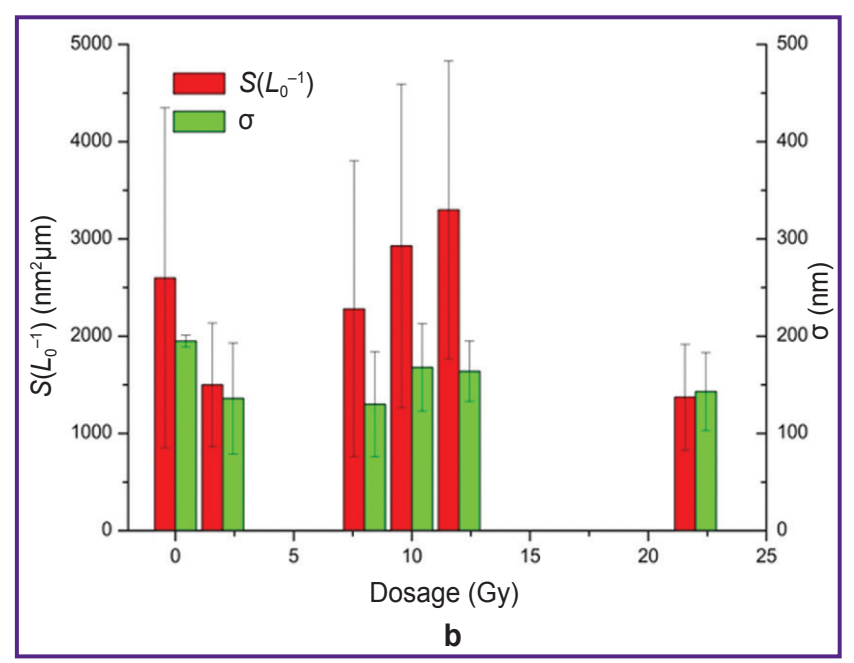

Figure 5. Flicker-noise spectroscopy parameterization of atomic force microscopy images obtained from the extracellular matrix of irradiated pelvic organs, demonstrating a unified character of changes in the extracellular matrix occurring after irradiation:

(a) bladder, (b) rectum, (c) skin; the sizes of the processed atomic force microscopy images are $6 \times 6 \mu \mathrm{m}$

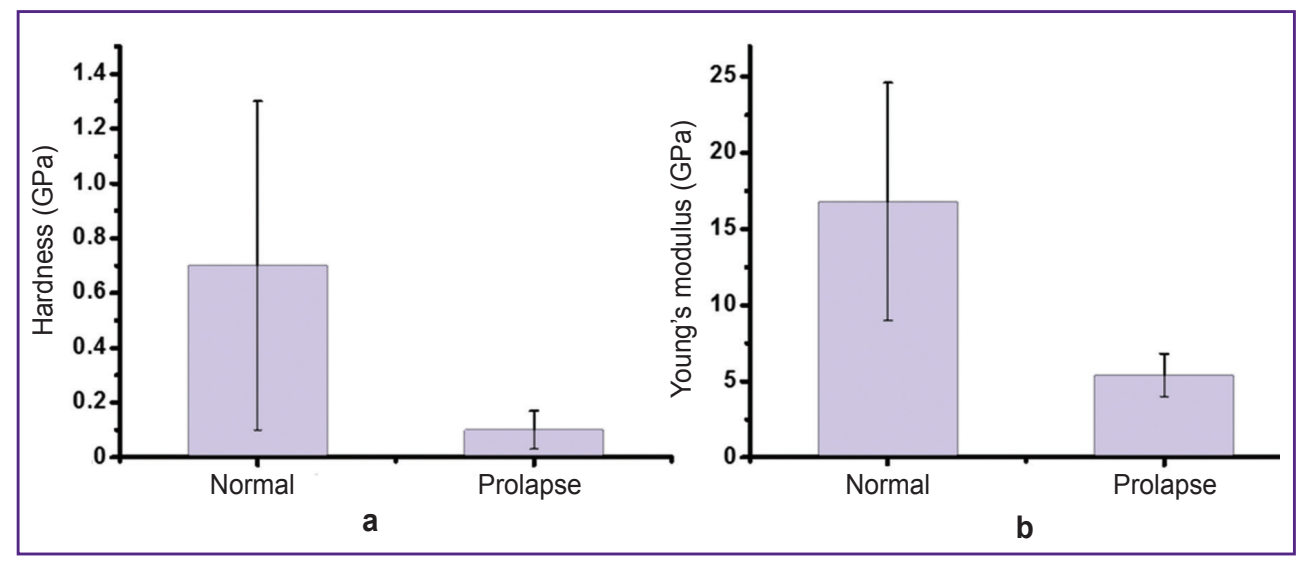

Figure 6. Nanoindentation study reveals deterioration of mechanical properties (hardness (a) and Young's modulus (b)) of the collagen fibrils bundles in the extracellular matrix of skin in patients with pelvic organ prolapse (from [17])

values of elastic moduli than the matrix with amianthoid transformation.

Consistency with the data of histological studies. In Figure 8, the typical results of histological studies are displayed, showing the same tendencies in the ECM transformation as those detected by AFM: the ECM disordering in the case of POP (a) and (b), the higher degree of disorganization in an atherosclerotic plaque 


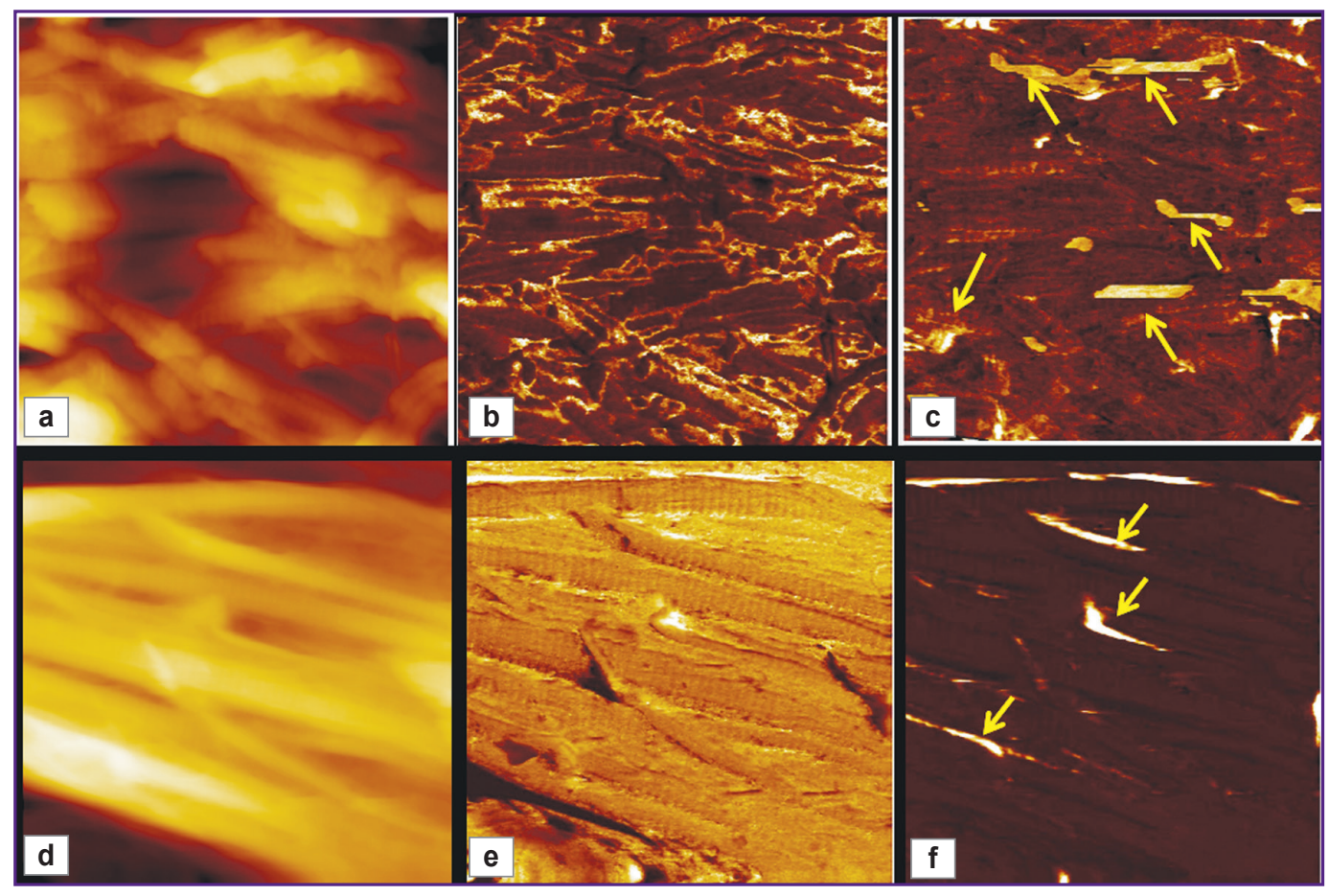

Figure 7. PeakForce QNM $^{\circledR}$ high-resolution images of the rib cartilage extracellular matrix Upper panel: (a) normal tissue, topography; (b) Young's modulus map; (c) adhesion map. Lower panel: (d) amianthoid transformation, topography; (e) Young's modulus map; (f) adhesion map; spots of the increased adhesion are marked by arrows. Scan sizes are $3 \times 3 \mu \mathrm{m}$

Figure 8. Typical histological studies of pathological processes in the connective tissue:

(a) normal skin; (b) skin of a POP patient; (c) atherosclerotic lesion Type Va; (d) atherosclerotic lesion Type Vc; FC - fibrous cap, LC lipid core; (e), (f) rectum: control samples (e) and samples 1 day after the 22 Gy treatment (f); black squares mark the regions of interest for the atomic force microscopy study. Van Gieson stain; magnification: $400 \times(a)$, (b); $10 \times$ (c), (d); $20 \times$ (e), (f)
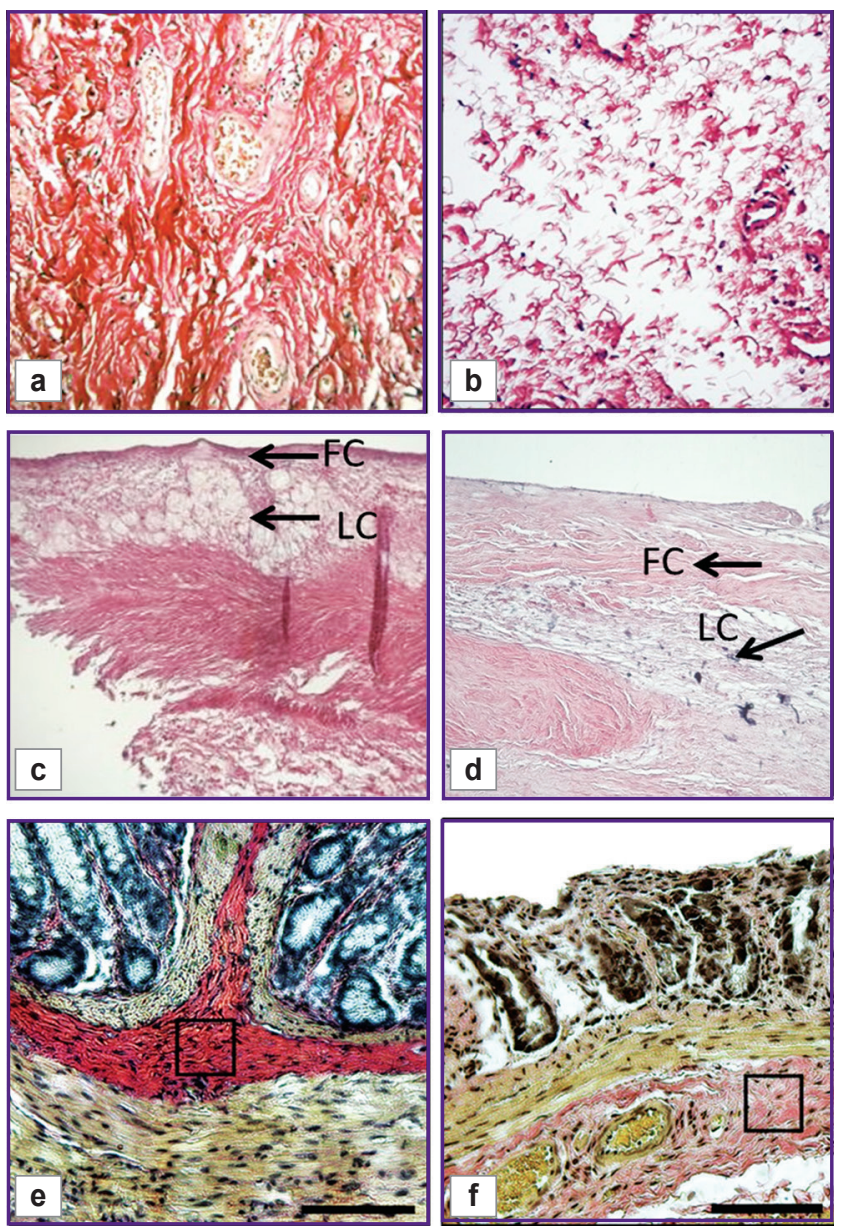
Type Va (c), as compared to that of an atherosclerotic plaque Type $V_{c}(d)$; the thickening and loosening of collagen fibers in the irradiated rectum (e) and (f). In the cases of atherosclerotic plaques and especially in the latter example, the changes in the collagen structures visualized by AFM are substantially more pronounced than those revealed by a conventional histological study using van Gieson's staining.

\section{Discussion}

Though it seems natural to study collagen-containing tissues under physiological conditions using AFM, and most of the AFM studies of such tissues are performed in fluid, it is hardly applicable for a quick assessment of a large number of specimens and also requires specific sample preparation, first of all, a proper tissue attachment to the support. The latter requirement may become a challenge, since swollen, gel-like tissue samples often do not attach firmly to a solid substrate, thus essentially impairing the AFM imaging quality.

In the last five years, we have applied AFM as a probe for the ECM changes related to a number of connective tissue conditions, with the purpose of developing a complementary ultrastructural method to conventional histological studies, which may utilize the same fixed, dry and sometimes even stained tissue sections. Here, we summarize the results of these studies (both completed ones and studies in progress) to demonstrate the feasibility of this approach.

AFM imaging of ECM excellently describes its structure since it allows visualization of collagen - the main ECM protein - at all the scales of its spatial arrangement in the ECM, from the nanostructure of collagen fibrils to the microstructure of collagen fibers and their bundles which form the ECM "skeleton" (see Figures 2, 3). For example, in our study of the ECM morphology in patients with POP [17], the AFM imaging showed pronounced deviations from the normal morphology both in the skin and in the pelvic ligament, at all the levels of collagen organization, showing the following morphological signs of the dysplastic changes leading to POP - loosening and separation of collagen fibers in the fiber bundles, fragmentation and disordering of collagen fibers and fibrils in the ECM of POP patients. In the study of the atherosclerosis in arterial walls [18], we found that the density of the 3D network of collagen fibers (and a subnetwork of fibrils) in the fibrotic layer of an atherosclerotic plaque visibly decreases with the progression of atherosclerosis. The AFM imaging also revealed drastic differences between the collagen packing of unstable (Type Va) and stable (Type Vc) fibrotic plaques. While a vulnerable (unstable) plaque has a loose and disordered packing both at the level of fibers and fibrils (apparently, responsible for its instability and propensity to rupture), a stable plaque has a significantly tighter packing. In the study of radiation-induced damage of pelvic organs, AFM visualized very early signs of the ECM destruction in the form of disordering of collagen fibers and fibrils inside them long before they could be revealed by optical microscopy. AFM tracks the time- and dose-dependent effects of radiation on the ECM, from the initial destruction of the protein matrix (characterized by disorganization and fragmentation of collagen fibers and fibrils) to the onset of the later fibrotic processes (manifested itself as bands of tightly packed parallel collagen fibers consisting of unidirectionally oriented fibrils). AFM imaging also allows visual evaluation of the content of non-fibrous material in the ECM. In our study of chondrosarcoma development, AFM has shown that malignancy is associated with an increased content of unstructured (non-collagenous) material in the ECM, proportionally to the degree of malignancy. All the above examples illustrate that AFM may probe and visualize essentially any structural ECM changes, occurring due to pathological processes of various causes, from the nanoscale to the microscale.

Quantification of the morphological features reflected in images is one of the greatest problems of imaging techniques. A concise numerical representation instead of a descriptive one may essentially simplify and validate visual morphological distinctions between samples. However, a satisfactory correlation between the appearance of an image and its metrological parameters is observed infrequently. Our AFM studies have shown that FNS may be successfully applied for this purpose. The rationale beyond our choice of the FNS parameterization instead of a standard metrological roughness (ISO 4287) lies in the fact that the surface of tissue sections has considerable height heterogeneity and, besides the features determined by the inner structure of a sample, reflects also the entire sample history from pre-treatment to drying to microtome sectioning. For such surfaces, the standard metrological parameters are generally not informative. In contrast, the FNS parameterization, via a careful analysis of frequencies in the power spectra along with the visual assessment of the corresponding images, allows elimination of the systematic artifacts and extraction of the sample's intrinsic structure parameters. We have managed to quantitatively describe the changing ECM morphology in a number of studies. In the POP study, we have shown that the FNS parameters notably change in POP as compared to the intact tissue, thus making FNS an additional diagnostic modality for dysplastic changes. Flicker-noise spectroscopy parameterization quantitatively confirmed the observed visual differences in the morphology of atherosclerotic plaques of different types. In the study of ionizing radiation-induced damage of pelvic organs, the FNS study not only confirmed the visual features of the ECM but also revealed a universal character of its changes for different types of connective tissue (bladder, rectum, skin).

The loss of the connective tissue ECM integrity, or the opposite process of ECM compaction due to fibrosis or accumulation of adhesive non-fibrous material, causes alteration of the ECM mechanical properties. Nanoindentation studies or imaging with the use of the 
PeakForce $\mathrm{QNM}^{\circledR}$ (Bruker, USA) allow detection of such changes. Our nanoindentation studies have demonstrated deterioration of the ECM mechanical properties in a tissue with a dysplastic process (POP study). The nanomechanical mapping provides not only the data on the distribution of Young's moduli over the ECM fragment but also mapping of the adhesive properties, simultaneously with the topography imaging and with the same high resolution. For example, in the study on amianthoid transformation of the rib cartilage, the PeakForce $\mathrm{QNM}^{\circledR}$ imaging detected dramatic alterations of the ECM topography, decrease of the Young's modulus and redistribution of glycosaminoglycans in the amianthoid transformation-altered ECM as compared to the native ECM.

All our AFM studies appeared in a good agreement with the corresponding histological findings and frequently appeared to have a superior sensitivity to pathologyrelated ECM rearrangements.

\section{Conclusions}

Our studies have shown that atomic force microscopy and related techniques may serve as excellent complementary tools to conventional histological studies of various pathological conditions of connective tissue. They provide valuable additional information on the connective tissue extracellular matrix destruction and remodeling, from micro- and nanoscale visualization of the collagen hierarchical structure to the numerical data on the extracellular matrix morphology to the nanomechanical properties of the extracellular matrix components.

Acknowledgements. The authors thank their collaborators in the above studies for providing the tissue samples and relevant clinical information Prof. A.B. Shekhter, Sechenov University, Moscow (POP, chondrosarcoma, and rib cartilage studies), Prof. N.D. Gladkova, Privolzhsky Research Medical University, Nizhny Novgorod (atherosclerosis study), Prof. A.V. Maslennikova, Privolzhsky Research Medical University, Nizhny Novgorod (radiation studies).

This study was financially supported by the Russian Foundation for Basic Research, Grant No.15-02-04505 (AFM, clinical studies), the Russian Science Foundation, Grant No.15-13-00140 (preparation of samples, histological studies), the Federal Agency of Scientific Organizations (Agreement No.007-GZ/Ch3363/26) in part concerning the materials and accessories, and the Sechenov University Grant (AFM, clinical studies).

Conflict of interests. The authors declare no conflict of interests.

\section{References}

1. Frantz C., Stewart K.M., Weaver V.M. The extracellular matrix at a glance. J Cell Sci 2010; 123(24): 4195-4200, https://doi.org/10.1242/jcs.023820.
2. Orgel J.P.R.O., Irving T.C., Miller A., Wess T.J. Microfibrillar structure of type I collagen in situ. Proc Natl Acad Sci USA 2006; 103(24): 9001-9005, https://doi.org/10.1073/ pnas.0502718103.

3. Kuznetsov S.S., Dudenkova V.V., Kochueva M.V., Kiseleva E.B., Ignatieva N.Yu., Zakharkina O.L., Sergeeva E.A., Babak K.V., Maslennikova A.V. Multiphoton microscopy in the study of morphological characteristics of radiation-induced injuries of the bladder. Sovremennye tehnologii $v$ medicine 2016; 8(2): 31-39, https://doi. org/10.17691/stm2016.8.2.04.

4. Mamalis A., Ho D., Jagdeo J. Optical coherence tomography imaging of normal, chronologically aged, photoaged and photodamaged skin. Dermatol Surg 2015; 41(9): 993-1005, https://doi.org/10.1097/dss.0000000000000457.

5. Maver U., Velnar T., Gaberšček M., Planinšek O., Finšgar M. Recent progressive use of atomic force microscopy in biomedical applications. Trends Analyt Chem 2016; 80: 96111, https://doi.org/10.1016/j.trac.2016.03.014.

6. Graham H.K., Hodson N.W., Hoyland J.A., MillwardSadler S.J., Garrod D., Scothern A., Griffiths C.E., Watson R.E., Cox T.R., Erler J.T., Trafford A.W., Sherratt M.J. Tissue section AFM: in situ ultrastructural imaging of native biomolecules. Matrix Biol 2010; 29(4): 254-260, https://doi. org/10.1016/j.matbio.2010.01.008.

7. Wen C.Y., Wu C.B., Tang B., Wang T., Yan C.H., Lu W.W., Pan H., Hu Y., Chiu K.Y. Collagen fibril stiffening in osteoarthritic cartilage of human beings revealed by atomic force microscopy. Osteoarthritis Cartilage 2012; 20(8): 916922, https://doi.org/10.1016/j.joca.2012.04.018.

8. Stolz M., Gottardi R., Raiteri R., Miot S., Martin I., Imer R., Staufer U., Raducanu A., Düggelin M., Baschong W., Daniels A.U., Friederich N.F., Aszodi A., Aebi U. Early detection of aging cartilage and osteoarthritis in mice and patient samples using atomic force microscopy. Nat Nanotechnol 2009; 4(3): 186-192, https://doi.org/10.1038/ nnano.2008.410.

9. Rigozzi S., Müller R., Stemmer A., Snedeker J.G. Tendon glycosaminoglycan proteoglycan sidechains promote collagen fibril sliding - AFM observations at the nanoscale. J Biomech 2013; 46(4): 813-818, https://doi.org/10.1016/j. jbiomech.2012.11.017.

10. Wallace J.M. Applications of atomic force microscopy for the assessment of nanoscale morphological and mechanical properties of bone. Bone 2012; 50(1): 420-427, https://doi.org/10.1016/j.bone.2011.11.008.

11. Sivasankar M., Ivanisevic A. Atomic force microscopy investigation of vocal fold collagen. Laryngoscope 2007; 117(10): 1876-1881, https://doi.org/10.1097/mlg.0b013e3180caa1df.

12. Lee S.J., Choi S., Kim M.S., Cheong Y., Kwak H.W., Park H.K., Jin K.H. Short-term effect of cryotherapy on human scleral tissue by atomic force microscopy. Scanning 2012; 35(5): 302-307, https://doi.org/10.1002/sca.21068.

13. Thomasy S.M., Raghunathan V.K., Winkler M., Reilly C.M., Sadeli A.R., Russell P., Jester J.V., Murphy C.J. Elastic modulus and collagen organization of the rabbit cornea: Epithelium to endothelium. Acta Biomaterialia 2014; 10(2): 785-791, https://doi.org/10.1016/j.actbio.2013.09.025.

14. Sridharan I., Ma Y., Kim T., Kobak W., Rotmensch J., Wang R. Structural and mechanical profiles of native collagen fibers in vaginal wall connective tissues. Biomaterials 2012; 33(5): 1520-1527, https://doi.org/10.1016/j.biomaterials.2011. 11.005. 


\section{BIOPHOTONICS IN REGENERATIVE MEDICINE}

15. Jorba I., Uriarte J.J., Campillo N., Farré R., Navajas D. Probing micromechanical properties of the extracellular matrix of soft tissues by atomic force microscopy. J Cell Physiol 2017; 232(1): 19-26, https://doi.org/10.1002/jcp.25420.

16. Kwok J., Grogan S., Meckes B., Arce F., Lal R., D'Lima D. Atomic force microscopy reveals age-dependent changes in nanomechanical properties of the extracellular matrix of native human menisci: implications for joint degeneration and osteoarthritis. Nanomedicine 2014; 10(8): 1777-1785, https://doi.org/10.1016/j.nano.2014.06.010.

17. Kotova S.L., Timashev P.S., Guller A.E., Shekhter A.B., Misurkin P.I., Bagratashvili V.N., Solovieva A.B. Collagen structure deterioration in the skin of patients with pelvic organ prolapse determined by atomic force microscopy. Microsc Microanal 2015; 21(2): 324-333, https://doi.org/10.1017/ s1431927615000148.

18. Timashev P.S., Kotova S.L., Belkova G.V.,
Gubar'kova E.V., Timofeeva L.B., Gladkova N.D., Solovieva A.B. Atomic force microscopy study of atherosclerosis progression in arterial walls. Microsc Microanal 2016; 22(2): 311-325, https://doi.org/10.1017/s1431927616000039.

19. Choy J.S., Mathieu-Costello O., Kassab G.S. The effect of fixation and histological preparation on coronary artery dimensions. Ann Biomed Eng 2005; 33(8): 1027-1033, https:// doi.org/10.1007/s10439-005-4854-4.

20. Dorph-Petersen K.-A., Nyengaard J.R., Gundersen H.J.G. Tissue shrinkage and unbiased stereological estimation of particle number and size. J Microsc 2001; 204(3): 232-246, https://doi.org/10.1046/j.1365-2818.2001.00958.x.

21. Mirsaidov U., Timashev S.F., Polyakov Y.S., Misurkin P.I., Musaev I., Polyakov S.V. Analytical method for parameterizing the random profile components of nanosurfaces imaged by atomic force microscopy. Analyst 2011; 136(3): 570-576, https://doi.org/10.1039/c0an00498g. 